\title{
Eye Movement Prediction by Kalman Filter with Integrated Linear Horizontal Oculomotor Plant Mechanical Model
}

\author{
Oleg V. Komogortsev \\ Department of Computer Science \\ Texas State University-San Marcos \\ ok11@txstate.edu
}

\author{
Javed I. Khan \\ Department of Computer Science \\ Kent State University \\ javed@kent.edu
}

\begin{abstract}
The goal of this paper is to predict future horizontal eye movement trajectories within a specified time interval. To achieve this goal a linear horizontal oculomotor plant mechanical model is developed. The model consists of the eye globe and two extraocular muscles: lateral and medial recti. The model accounts for such anatomical properties of the eye as muscle location, elasticity, viscosity, eyeglobe rotational inertia, muscle active state tension, length tension and force velocity relationships. The mathematical equations describing the oculomotor plant mechanical model are transformed into a Kalman filter form. Such transformation provides continuous eye movement prediction with a high degree of accuracy. The model was tested with 21 subjects and three multimedia files. Practical application of this model lies with direct eye gaze input and interactive displays systems as a method to compensate for detection, transmission and processing delays.
\end{abstract}

CR Categories: I.6.4 [Simulation and Modeling]: Model Validation and Analysis; J.7 [Computers in Other Systems]: Process control, Real time.

Keywords: Eye movement prediction, oculomotor plant, Kalman filter, human computer interaction.

\section{Introduction}

Direct eye-gaze input [Jacob 1995; Murtagh et al 2002; Komogortsev 2007a] and interactive display systems are prone to delays. The delays are comprised of detection, processing and transmission times. Depending on the specific case the delays can reduce a system's performance and introduce interaction errors. Eye movement prediction is one of the ways to compensate for the delay effects.

We explored the idea of the eye movement prediction in our previous work [Komogortsev 2007a], a live system was created [Komogortsev 2007b] using this concept for delay compensation. In that system the vertical and horizontal eye movement component was predicted by a Two State Kalman Filter (TSKF), two states being eye position and velocity. This paper improves the accuracy of the eye movement prediction in the horizontal plane by building a linear Oculomotor Plant Mechanical Model (OPMM). Oculomotor plant is defined here as an eye globe plus extraocular muscles. The model is adopted from Bahil's work [Bahill 1980]. There are two major contributions added to the model a) the ability

Copyright $(\subset) 2008$ by the Association for Computing Machinery, Inc.

Permission to make digital or hard copies of part or all of this work for personal or classroom use is granted without fee provided that copies are not made or distributed for commercial advantage and that copies bear this notice and the full citation on the first page. Copyrights for components of this work owned by others than ACM must be honored. Abstracting with credit is permitted. To copy otherwise, to republish, to post on servers, or to redistribute to lists, requires prior specific permission and/or a fee. Request permissions from Permissions Dept, ACM Inc., fax +1 (212) 869-0481 or e-mail permissions@acm.org.

ETRA 2008, Savannah, Georgia, March 26-28, 2008.

(C) 2008 ACM 978-1-59593-982-1/08/0003 $\$ 5.00$ to start a saccade from any eye location b) the ability to make saccade in any direction in the horizontal plane. The mathematical equations describing the OPMM are transformed into a Kalman filter form that continuously predicts the eye movement signal. Kalman filter is selected because of its ability to minimize the error between the measured and the estimated eye position [Brown and Hwang 1997] and detect the onset of saccades [Sauter 1991]. We call the resulting model Oculomotor Plant Kalman Filter (OPKF). The OPKF has six states that consist of: angular eye position, velocity, muscles' locations, and muscles' forces.

Our simulation results that consisted of 21 subjects and three test multimedia files show a $18-28 \%$ of increase in accuracy of prediction during saccades and $7-13 \%$ increase overall when OPKF model is used instead of the TSKF model.

\section{Human Visual System}

Our vision is provided by our eyes. There are three major eye movement types: fixations, saccades and pursuits [Duchowski 2003], that move our eyes around and provide various quality of vision. The eye movement prediction model should be capable of predicting the eye movement signal during all eye movements, therefore the goal of this section is to describe mechanical elements modeling the anatomical structure of the eye and provide mathematical equations describing oculomotor plant during various eye movement types.

\subsection{Extraocular Muscles \& Neuronal Control}

The eye globe rotates in its socket through the use of six muscles. These six muscles are: the medial and the lateral recti - the muscles responsible for horizontal eye movements; superior and inferior recti - the muscles responsible for vertical eye movements; superior and inferior oblique - the muscles responsible for eye rotations around its primary axis of sight; and vertical eye movements. The brain sends a neuronal control signal to each muscle to direct the muscle to perform its work. A neuronal control signal is anatomically implemented as a neuronal discharge that is sent through a nerve to a designated muscle from a specific part of the brain [Sparks 2002]. The frequency of this discharge determines the level of muscle innervation and results in a specific amount of work that a muscle can perform. During saccades the neuronal control signal for each muscle resembles a pulse-step function [Bahill 1980]. The eye position during the onset of a saccade, the saccade's amplitude and direction define pulse and step parameters of the control signal. During eye fixations neuronal discharge is performed at a constant rate that is linearly related to the eye position. 


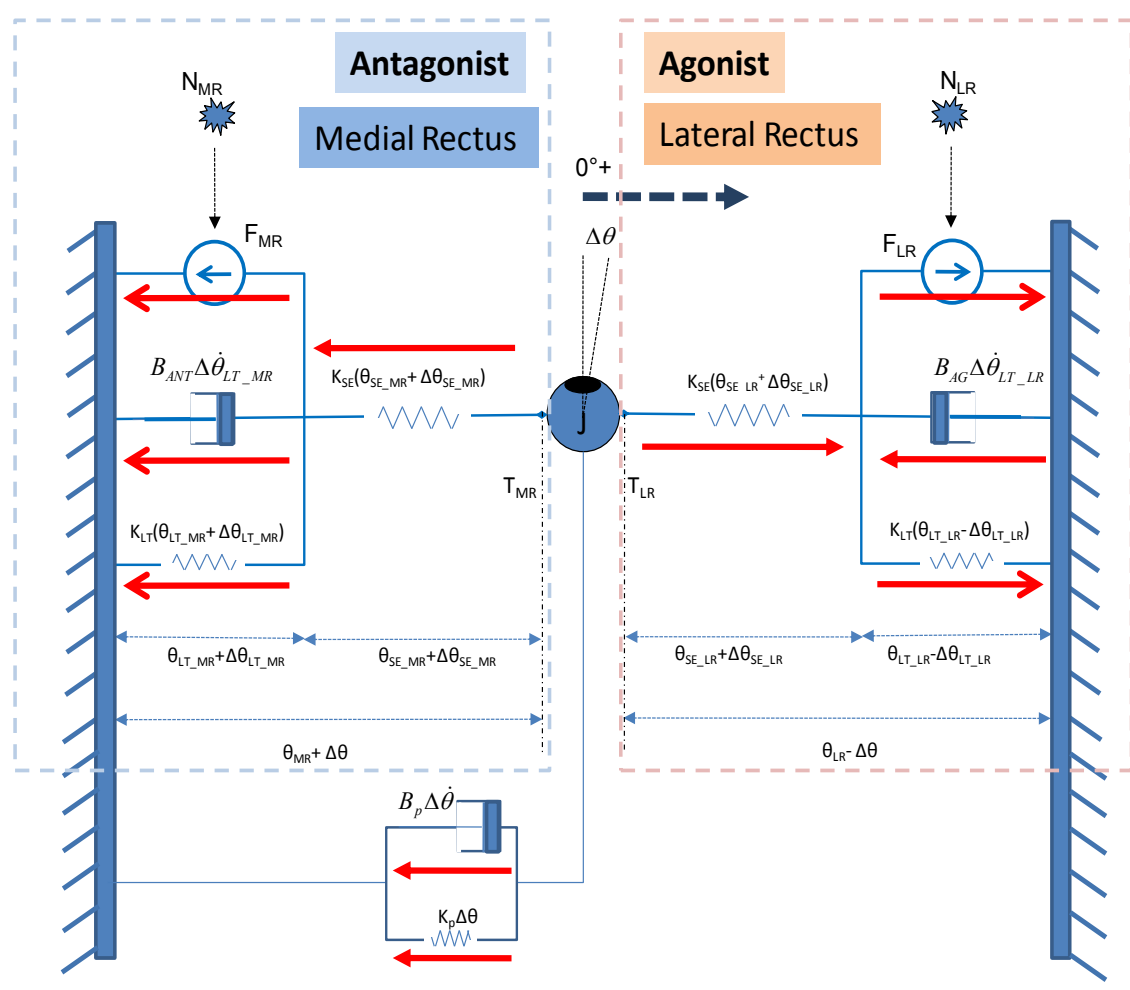

Figure 1. The Oculomotor Plant Mechanical Model employed for positive amplitude saccades. Arrows show the direction of forces for each component. $\Delta \theta-$ eye rotation. $\mathrm{J}$ is the rotational inertia of the eye globe.

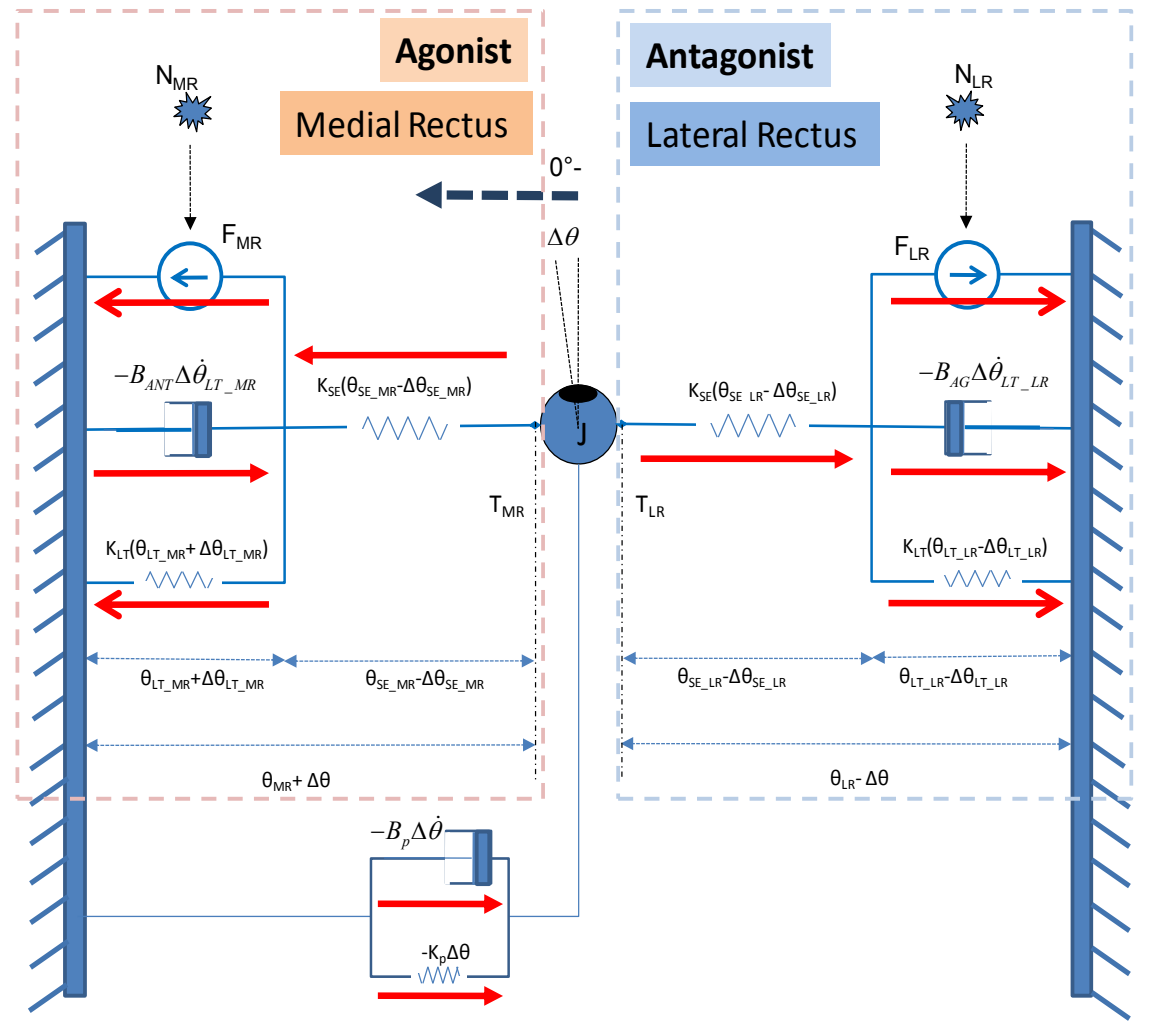

Figure 2. The Oculomotor Plant Mechanical Model employed for negative amplitude saccades.

\section{2 Model \\ Oculomotor Plant Mechanical}

The OPMM developed in this paper represents the anatomy of the right eye, though the model can be easily modified to work for the left eye as well. Positive amplitude saccades are defined as saccades that move the right eye rightwards. Such saccades are performed by the lateral rectus (the muscle that is closer to the ear) as the agonist and by the medial rectus (the muscle that is closer to the nose) as the antagonist. The agonist muscle pulls the eye globe in the required direction and the antagonist muscle resists the pull. Negative amplitude saccades are defined as saccades that move the right eye leftwards, they are executed by the lateral rectus as the antagonist and by the medial rectus as the agonist. The antagonist's muscle parameters are identified with $\mathrm{AG}$ subscript (example $\mathrm{N}_{\mathrm{AG}}, \mathrm{B}_{\mathrm{AG}}$ ), antagonist with ANT, lateral rectus with LR and medial rectus with MR. Note that either the lateral or the medial recti can play the role of the agonist or the antagonist. Parameters without those subscripts are identical to both types of muscles. The eye globe's radius is assumed to be $11 \mathrm{~mm}$.

We present the neuronal control signal values and force equations necessary to generate fixations and saccades of the negative and positive amplitudes. The lateral and the medial recti are modeled through a system of mechanical components mimicking the anatomical properties of a muscle.

\subsubsection{Muscle Properties}

A muscle is a very complex structure [Wilkie 1976]. The Muscle Mechanical Model (MMM) can be represented through several components. These components are the following: passive elasticity, active state tension, a series elasticity, a length-tension component and a force velocity relationship [Bahill 1980; Collins 1975; Robinson et al. 1969].

Passive Elasticity: Each body muscle in the rest state is elastic. The rested muscle can be stretched by applying force. The muscle extension is proportional to the force applied. The passive elasticity results largely from the meshwork of connective tissue within the muscle, whose fibers become progressively taut when the muscle is stretched [Wilkie 1976]. The passive muscle component is non linear, but in this paper it is modeled as an ideal linear spring. The numerical value for the spring coefficient representing passive elasticity was estimated by Collins [Collins 1975] to be $K_{p}=0.5$ grams of tension per degree. 
Active State Tension: Each muscle produces active state tension when it is stimulated. If stimulated by a single wave of neurons a muscle twitches then relaxes. A muscle goes into the tetanic state, when it is stimulated by neurons at a specific frequency continuously [Wilkie 1976]. The frequency of the neuronal discharge is determined by the brain, depending of the type of the eye movement and eye position. When a tetanic stimulation occurs, a muscle develops tension, trying to contract. The resulting tension is called the active state tension. The intensity of the active state tension depends upon the frequency of the neuronal discharge. An ideal force generator component is used in the MMM to represent active state tension as $F_{L R}$ for lateral rectus and as $\mathrm{F}_{\mathrm{MR}}$ for medial rectus

Length Tension Relationship: The tension that a muscle develops as a result of neuronal stimulation partially depends on its length. Usually the tension in a significantly contracted muscle is less than the maximum tension that a muscle is capable of creating at its optimal length. A length tension effect occurs due to the following reasons: when a stimulated muscle is lengthened considerably, the area of overlap of thick and thin filaments (anatomical components responsible for muscle contraction) diminishes and as a result the muscle's active state tension diminishes as well. When a stimulated muscle is shortened considerably the thin and thick filaments interfere with the series elastic elements of the muscle, absorbing part of the tension developed, thus reducing the overall active state tension. Additionally when a muscle is significantly shortened, neuronal stimulation does not reach inner muscle contractile fibers [Wilkie 1976]. Each of these phenomena contributes to a non linear relationship between the tension and length. Nonetheless, in this paper length tension relationship is modeled as an ideal linear spring. The linear coefficient $\mathrm{K}_{\mathrm{LE}}$ of the spring was measured by Bahill [Bahill 1980] to be 1.2 grams of tension per degree.

Series Elasticity: The series elasticity is in series with the active force generator, hence the name. Anatomically, spring elasticity components are located on the tendon and in myosin and in the actin bridges of the muscle fibers. In the MMM series elasticity component is modeled as an ideal linear spring. The linear coefficient $\mathrm{K}_{\mathrm{SE}}$ of the spring was measured by Collins [Collins 1975 ] to be 2.5 grams of tension per degree.

Force Velocity Relationship: This relationship shows that a muscle is capable of producing larger forces at lower velocities. This dependency of force upon velocity varies for different levels of a neuronal control signal and depends on whether a muscle shortens or being stretched. It is believed that the force velocity relationship exists due to the different rates of chemical reactions inside a muscle at various length-changing velocities [Wilkie 1976]. Bahill suggests using linear dashpots $\mathrm{B}_{\mathrm{AG}}$ for the agonist muscle and $\mathrm{B}_{\mathrm{ANT}}$ for the antagonist muscle [Bahill 1980]. In our model, the velocity of muscle contraction is connected to a change of length in the length tension component of the muscle and represented by the following variables: $\Delta \dot{\theta}_{\mathrm{LT} \_\mathrm{LR}}-$ lateral rectus, $\Delta \dot{\theta}_{\mathrm{LT} \_\mathrm{MR}}-$ medial rectus.

\subsection{Saccades of the positive amplitude}

Saccades of the positive amplitude are performed by the OPMM presented in Figure 1. The eye is rotating by the distance of $\Delta \theta$ degrees. Prior to a saccade the displacement (distance from its equilibrium length) inside of the of series elasticity component of lateral rectus is $\theta_{\mathrm{SE}_{-} \mathrm{LR}}$, and legth tension component $\theta_{\mathrm{LT} \_\mathrm{LR}}$.
Therefore the total displacement inside of the lateral rectus is $\theta_{\mathrm{LR}}=\theta_{\mathrm{SE} \_ \text {LR }}+\theta_{\mathrm{LT}}$ LR . Similar logic applies to the medial rectus.

\subsubsection{Agonist Muscle Mechanical Model of Lateral Rectus}

Right part of Figure 1 shows neronal firing inducing the contraction of the lateral rectus by generating the active state tension. During contraction the length tension component shortens by $\Delta \theta_{\mathrm{LT}_{-} \mathrm{LR}}$, the series elasticity component lengthens by $\Delta \theta_{\mathrm{SE}_{-} \mathrm{LR}}$, resulting in the eye rotation of $\Delta \theta$ degrees.

Forces that work inside of the agonist MMM during eye rotation can be broken into two force groups. The first group consists of the active state tension $\mathrm{F}_{\mathrm{LR}}$; length tension force $K_{L T}\left(\theta_{L T_{-} L R}-\Delta \theta_{L T-L R}\right)$, working in the same direction as $\mathrm{F}_{\mathrm{LR}}$; damping force $-B_{A G} \Delta \dot{\theta}_{L T_{-} L R}$, accounting for the force velocity relationship resists the contraction of lateral rectus. Note that the amount of resistive force produced by the damping component is based upon the velocity of contraction of the length tension $\Delta \dot{\theta}_{L T_{-} L R}$. Summing the forces of the first group we get following equation:

$$
T_{L R}=F_{L R}+K_{L T}\left(\theta_{L T_{-} L R}-\Delta \theta_{L T_{-} L R}\right)-B_{A G} \Delta \dot{\theta}_{L T_{-} L R}
$$

The second force group consists of the series elasticity component that propagates force $T_{L R}$, generated by the first group, to the eye globe. Series elasticity component is a linear spring therefore $T_{L R}$ can be computed as:

$$
T_{L R}=K_{S E}\left(\theta_{S E_{-} L R}+\Delta \theta_{S E_{-} L R}\right)
$$

Equations 1 and 2 can be rearranged in a form that calculates force $\mathrm{T}_{\mathrm{LR}}$ in terms of the eye rotation $\Delta \theta$, and displacement $\Delta \theta_{L T_{-} L R}$.

$$
\begin{gathered}
T_{L R}=\frac{\hat{F}_{L R} K_{S E}}{K_{S E}+K_{L T}}-\frac{\Delta \theta K_{S E} K_{L T}}{K_{S E}+K_{L T}}-\hat{B}_{A G} \Delta \dot{\theta}_{L T_{-} L R} \\
T_{L R}=K_{S E}\left(\Delta \theta_{L T_{-} L R}-\Delta \theta\right)
\end{gathered}
$$

where $\hat{F}_{L R}=F_{L R}-K_{S E}\left(\theta_{L R}-\theta_{L T_{L} L R}\right)+K_{L T} \theta_{L T_{-} L R}$ The detailed calculations can be found in [Komogortsev $2007 \mathrm{c}$ ].

\subsubsection{Antagonist Muscle Mechanical Model of Medial Rectus}

Left part of Figure 1 presents the model. Medial rectus gets extended by the pull of the lateral rectus. Length tension component extends by $\Delta \theta_{\mathrm{LT}}$ MR and the series elasticity component extends by $\Delta \theta_{\mathrm{SE} \text { MR }}$.

Two groups of muslce forces similar to the agonist case are present. The first group consists of the active state tension $-\mathrm{F}_{\mathrm{MR}}$, resisting the agonist pull; length tension force $-K_{L T}\left(\theta_{L T-M R}+\right.$ $\left.\Delta \theta_{L T_{-} M R}\right)$, resisting the agonist pull; damping force $-B_{A N T} \Delta \dot{\theta}_{L T_{-} M R}$, accounting for the force velocity relationship, resists the lengthening of the medial rectus. Summing the forces of the first group we get following equation:

$$
T_{M R}=-F_{M R}-K_{L T}\left(\theta_{L T_{-} M R}-\Delta \theta_{L T_{-} M R}\right)-B_{A N T} \Delta \dot{\theta}_{L T_{-} M R}
$$

$T_{M R}$ can be also computed through the properties of the series elasticity component.

$$
T_{M R}=-K_{S E}\left(\theta_{S E_{-} M R}+\Delta \theta_{S E_{-} M R}\right)
$$

Equations 5 and 6 can be rearranged in a form that calculates force $\mathrm{T}_{\mathrm{MR}}$ in terms of the eye rotation $\Delta \theta$, and displacement $\Delta \theta_{L T_{-} M R}$.

$$
\begin{gathered}
T_{M R}=-\frac{\hat{F}_{M R} K_{S E}}{K_{S E}+K_{L T}}-\frac{\Delta \theta K_{S E} K_{L T}}{K_{S E}+K_{L T}}-\hat{B}_{A G} \Delta \dot{\theta}_{L T_{-} M R} \\
T_{M R}=-K_{S E}\left(\Delta \theta-\Delta \theta_{L T_{\_} M R}\right)
\end{gathered}
$$

where $\hat{F}_{M R}=F_{M R}-K_{S E}\left(\theta_{M R}-\theta_{L T \_M R}\right)+K_{L T} \theta_{L T \_M R}$. 


\subsection{Saccades of the negative amplitude}

Saccades of the negative amplitude are performed by a mechanical model presented in Figure 2.

\subsubsection{Agonist Muscle Mechanical Model of Medial Rectus}

Left part of Figure 2 shows neronal firing inducing the contraction of the medial rectus by generating the active state tension. During contraction the length tension component shortens by $\Delta \theta_{\mathrm{LT} \text { MR }}$, the series elasticity component lengthens by $\Delta \theta_{\mathrm{SE} \_\mathrm{MR}}$, resulting in the eye rotation of $\Delta \theta$ degrees. Note that values of $\Delta \theta_{\mathrm{LT} \_\mathrm{MR}}, \Delta \theta_{\mathrm{SE}_{-} \mathrm{MR}}$, $\Delta \theta$ have negative sign.

Using similar logic outlined for positive amplitude saccades and using the diagram represented by Figure 2 we can can write the equation accounting for active state tension, length tension and damping force inside of the lateral rectus:

$$
T_{M R}=-F_{M R}-K_{L T}\left(\theta_{L T_{-} M R}+\Delta \theta_{L T_{-} M R}\right)+\left(-B_{A G} \Delta \dot{\theta}_{L T_{-} M R}\right) \quad \mathbf{9}
$$

$\mathrm{T}_{\mathrm{MR}}$ value can be calculated by the properties of the series elasticity component as:

$$
T_{M R}=-K_{S E}\left(\theta_{S E_{-} M R}-\Delta \theta_{S E_{-} M R}\right)
$$

Equations 9 and 10 can be rearranged in a form that calculates force $\mathrm{T}_{\mathrm{MR}}$ in terms of the eye rotation $\Delta \theta$, and displacement $\Delta \theta_{L T \_M R}$.

$$
\begin{gathered}
T_{M R}=\frac{\hat{F}_{M R} K_{S E}}{K_{S E}+K_{L T}}+\frac{\Delta \theta K_{S E} K_{L T}}{K_{S E}+K_{L T}}+\hat{B}_{A G} \Delta \dot{\theta}_{L T_{-} M R} \\
T_{M R}=K_{S E}\left(\Delta \theta-\Delta \theta_{L T_{-} M R}\right)
\end{gathered}
$$

\subsubsection{Antagonist Muscle Mechanical Model of Lateral Rectus}

Using part of the Figure 2 presenting the antagonist muscle we can sum the forces of the first group:

$$
T_{L R}=F_{L R}+K_{L T}\left(\theta_{L T_{-} L R}-\Delta \theta_{L T_{-} L R}\right)+\left(-B_{A N T} \Delta \dot{\theta}_{L T_{-} L R}\right) \quad \mathbf{1 3}
$$

$\mathrm{T}_{\mathrm{LR}}$ can be also calculated as:

$$
T_{L R}=K_{S E}\left(\theta_{S E_{-} L R}-\Delta \theta_{S E_{-} L R}\right)
$$

Equations 13 and 14 allow to calculating the force $T_{M R}$ in terms of the eye rotation $\Delta \theta$, and displacement $\Delta \theta_{L T} L R$.

$$
\begin{gathered}
T_{L R}=\frac{\hat{F}_{L R} K_{S E}}{K_{S E}+K_{L T}}-\frac{\Delta \theta K_{S E} K_{L T}}{K_{S E}+K_{L T}}-\hat{B}_{A N T} \Delta \dot{\theta}_{L T_{-} L R} \\
T_{L R}=K_{S E}\left(\Delta \theta_{L T_{-} L R}-\Delta \theta\right)
\end{gathered}
$$

16

\subsection{Neuronal Control Signal}

\subsubsection{Fixations}

During a fixation state the active state tension $\hat{F}_{L R}, \hat{F}_{M R}$ are assumed to be same as the neuronal control signal sent to the muscle [Bahill 1980]. Using measured values of forces $T_{L R}, T_{M R}$ Bahill has calculated the neuronal control signal values during the eye fixation as:

$$
\begin{aligned}
& N_{A G_{-} f i x}(\Delta \theta)=(20.6+2.37|\Delta \theta|) \\
& N_{A N T_{-} f i x}(\Delta \theta)=\left\{\begin{array}{c}
(20.6-0.74|\Delta \theta|) \text { grams if }|\Delta \theta| \leq 28^{\circ} \\
0 \text { grams if }|\Delta \theta|>28^{\circ}
\end{array}\right.
\end{aligned}
$$

$|\Delta \theta|$ is the absolute value of $\Delta \theta$.

\subsubsection{Saccades}

Each saccade is generated by a neuronal control signal that looks like a pulse step function [Robinson 1969]. This signal can be presented by the following equations:

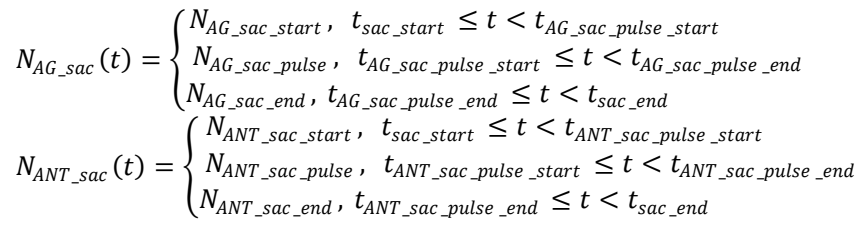
$\mathrm{t}_{\text {name }}$ constants present time parameters for each type of muscle and action phase. $\mathrm{t}$ is the time elapsed from the beginning of the saccade. The OPMM developed in this paper uses the time constants values presented below.

$t_{\text {sac_start }}=0$,

$t_{\text {sac_end }}=\left(2.2 *\left|\theta_{\text {sac_amp }}\right|+21\right)$ msec.,

$\theta_{\text {sac_amp }}$ is the amplitude of the saccade measured in degrees,

The agonist and antagonist muscle related time constants are:

$t_{A G_{-} s a c_{\text {_pulse_start }}}=t_{\text {sac_start }}+3$ msec.

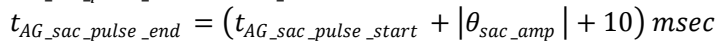

$t_{\text {ANT_sac_pulse_start }}=t_{\text {sac_start }_{\text {_t }} \text {, }}$

$t_{\text {ANT_sac_pulse_end }}=\left(t_{\text {AG_sac_pulse_start }}+\left|\theta_{\text {sac_amp }}\right|+16\right)$ msec .

The value representing the width of the antagonist pulse $\left(t_{A N T_{-} s a c_{-} p u l s e \_e n d}-t_{A N T_{\text {_sac_pulse_start }}}\right)$ is selected as a result of physiological measurements that indicated that the agonist pulse starts $3 \mathrm{msec}$. after the start of the antagonist pulse and ends 3 msec. before the end of the antagonist pulse.

In live system a Kalman Filter detects future saccade's parameters by a mechanism presented in our previous work [Komogortsev 2007b]. The parameters such as amplitude, the eye position at beginning and the end of the saccade should be supplied to the OPMM in terms of neuronal control signal. Therefore, we have created the functions that transform saccade parameters into neuronal control signal: $N_{A G_{-} s a c_{s} s t a r t}, N_{A N T_{\text {_ssac_start }}}, N_{A G_{-} s a c \_e n d}$, $N_{A N T_{\_} s a c_{\_} \text {end }}$.

$N_{A G_{-} \text {sac_start }}\left(\theta_{\text {sac_start }}\right)$

$=\left\{\begin{array}{l}N_{A G_{-} F I X}\left(\theta_{\text {sac_start }}\right), \quad \text { if agonist prior to saccade } \\ N_{A N T_{F I X}}\left(\theta_{\left.\text {sac }_{\text {start }}\right),}\right) \quad \text { if antagonist prior to saccade }\end{array}\right.$

$N_{A G_{\text {sac_palse }}}\left(\theta_{\text {sac_amp }}\right)= \begin{cases}55+11\left|\theta_{\text {sac_amp }}\right|, & \text { if } \theta_{\text {sac_amp }} \leq 11^{\circ} \\ 160+2\left|\theta_{\text {sac_amp }}\right|, & \text { if } \theta_{\text {sac_amp }}>11^{\circ}\end{cases}$

$N_{A G_{\text {_asa__end }}}\left(\theta_{\text {sac_end }}\right)$

$=\left\{\begin{array}{c}N_{A G_{-} F I X}\left(\theta_{\text {sac_end }}\right), \quad \text { if agonist after saccade } \\ N_{A N T_{-} F I X}\left(\theta_{\text {sac_end }}\right), \quad \text { if antagonist after saccade }\end{array}\right.$

$N_{\text {ANT_sac_start }}\left(\theta_{\text {sac_start }}\right)$

$=\left\{\begin{array}{c}N_{A G_{-} F I X}\left(\theta_{\text {sac_start }}\right), \text { if agonist prior to saccade } \\ N_{A N T_{-} F I X}\left(\theta_{\text {sac_start }}\right), \text { if antagonist prior to saccade }\end{array}\right.$

$N_{\text {ANT_sac_pulse }}\left(\theta_{\text {sac_amp }}\right)=0.5$

$N_{\text {ANT_sac_end }}\left(\theta_{\text {sac_end }}\right)$

$=\left\{\begin{array}{c}N_{A G_{-} F I X}\left(\theta_{\text {sac_end }}\right), \quad \text { if agonist after saccade } \\ N_{A N T_{-} F I X}\left(\theta_{\text {sac_end }}\right), \quad \text { if antagonist after saccade }\end{array}\right.$

The values for $N_{A G_{-} s a c_{-} p u l s e}, N_{A N T_{\text {_s }} a c_{-} p u l s e}$ and time constants except $t_{\text {sac_start }}, t_{\text {sac_end }}$ were taken from Bahill's work [Bahill 1980].

\subsubsection{Pursuits}

Neuronal control signal during pursuit eye movements is not defined for the OPMM presented in this paper. Eye movement prediction during pursuits is solely done by the Two State Kalman Filter.

\subsection{Active State Tension}

Though the neuronal control signal $N_{A G_{-} s a c}(t)$ and $N_{A N T_{-} s a c}(t)$, rises and drops instantaneously, neither the forces that muscles apply to the eye globe nor active state tensions rise to their 
maximum values immediately. This happens due to the anatomical characteristics of the neuronal signaling [Bahill 1980]. In the proposed model, the active state tension is a result of a low pass filtering process performed upon the neuronal control signal. Active state tension dynamics can be represented with the following differential equations at each time interval

$$
\begin{gathered}
\dot{\hat{F}}_{A G}(t)=\frac{N_{A G_{-} s a c}-\hat{F}_{A G}(t)}{\tau_{A G_{\_} s a c}} \\
\dot{\hat{F}}_{A N T}(t)=\frac{N_{A N T_{-} s a c}-\hat{F}_{A N T}(t)}{\tau_{A N T_{\_} s a c}}
\end{gathered}
$$

$\tau_{A G_{-} s a c}$ and $\tau_{A N T_{\text {_sac }}}$ are functions that define the low pass filtering process; they are defined by the activation and deactivation time constants.

All presented activation/deactivation time constants are selected empirically to match human physiological data [Bahill 1980].

\subsection{Oculomotor Plant Mechanical Model Equations}

\subsubsection{Positive amplitude saccades Figure 1}

The lateral rectus as the agonist applies the force to the eye globe that can be calculated by Equations 3 and 4. Those equations can be combined together:

$$
K_{S E}\left(\Delta \theta_{L T_{-} L R}-\Delta \theta\right)=\frac{\hat{F}_{L R} K_{S E}}{K_{S E}+K_{L T}}-\frac{\Delta \theta K_{S E} K_{L T}}{K_{S E}+K_{L T}}-\hat{B}_{A G} \Delta \dot{\theta}_{L T_{-} L R}
$$

The medial rectus as the antagonist applies the force to the eye globe that can be calculated by Equations 7 and 8 . Those equations can be combined together:

$$
K_{S E}\left(\Delta \theta-\Delta \theta_{L T \_M R}\right)=\frac{\hat{F}_{M R} K_{S E}}{K_{S E}+K_{L T}}+\frac{\Delta \theta K_{S E} K_{L T}}{K_{S E}+K_{L T}}+\hat{B}_{A N T} \Delta \dot{\theta}_{L T_{-} M R}
$$

Applying Newton's second law, the sum of all forces acting on the eye globe equals the acceleration of the eye globe multiplied by the inertia of the eye globe.

$$
J \Delta \ddot{\theta}=T_{L R}-T_{M R}-K_{p} \Delta \theta-B_{p} \Delta \dot{\theta}
$$

$\mathrm{J}=0.000043$ grams-s ${ }^{2} /$ degrees - eye globe's inertia, $\Delta \theta$ - eye rotation, $\Delta \dot{\theta}$ velocity of the eye rotation, $\Delta \ddot{\theta}$ eye rotation acceleration, $\mathrm{B}_{\mathrm{p}}=0.06$ grams-s/degrees - viscosity of the tissues around the eye globe. $T_{L R}$ can be calculated by Equation 4 and $T_{M R}$ can be calculated by Equation 8. Thus Equation 21 can be transformed into:

$$
J \Delta \ddot{\theta}=K_{S E}\left(\Delta \theta_{L T_{-} L R}-\Delta \theta\right)-K_{S E}\left(\Delta \theta-\Delta \theta_{L T_{-} M R}\right)-K_{p} \Delta \theta-B_{p} \Delta \dot{\theta}
$$

Equations - 17,18,19,20,22 are five differential equations with six variables $\left(\Delta \theta, \Delta \theta_{L T_{-} L R}, \Delta \theta_{L T_{\_} M R}, \Delta \dot{\theta}, \hat{F}_{L R}, \hat{F}_{M R}\right)$ A sixth differential equation can be added as:

$$
\Delta \dot{\theta}=\Delta \dot{\theta}
$$

These six differential equations completely describe the OPMM during saccades of the positive amplitude.

\subsubsection{Negative amplitude saccades Figure 2}

The logic presented in the previous section employed in deriving mathematical equations for saccades of negative amplitude [Komogortsev 2007c]:

$$
\begin{gathered}
K_{S E}\left(\Delta \theta_{L T_{-} L R}-\Delta \theta\right)=\frac{\hat{F}_{L R} K_{S E}}{K_{S E}+K_{L T}}-\frac{\Delta \theta K_{S E} K_{L T}}{K_{S E}+K_{L T}}-\hat{B}_{A N T} \Delta \dot{\theta}_{L T_{-} L R} \\
K_{S E}\left(\Delta \theta-\Delta \theta_{L T_{-} M R}\right)=\frac{\hat{F}_{M R} K_{S E}}{K_{S E}+K_{L T}}+\frac{\Delta \theta K_{S E} K_{L T}}{K_{S E}+K_{L T}}+\hat{B}_{A G} \Delta \dot{\theta}_{L T_{-} M R} \\
J \Delta \ddot{\theta}=K_{S E}\left(\Delta \theta_{L T_{-} L R}-\Delta \theta\right)-K_{S E}\left(\Delta \theta-\Delta \theta_{L T_{-} M R}\right)+K_{p} \Delta \theta+B_{p} \Delta \dot{\theta}
\end{gathered}
$$

Equations $17,18,24,25,26$ augmented by the identity $\Delta \dot{\theta}=\Delta \dot{\theta}$ describe the system completely.
It is remarkable to note that Equation 19, representing the force dynamics of the lateral rectus, is almost the same as Equation 24, except the value of the dashpot coefficient. The same fact can be noted about the Equation 20 and Equation 25. This result indicates that the mechanical dynamics remain essentially the same for each muscle independent of the role this muscle plays during a saccade The equation mapping all forces acting on the eye globe to the eye acceleration and inertia during a saccade is the same for the saccades of both negative and positive amplitudes.

\section{Oculomotor Plant Kalman Filter}

\subsection{Basics of Kalman Filtering}

The Kalman filter is a recursive estimator that computes a future estimate of the dynamic system state from a series of incomplete and noisy measurements. A Kalman Filter minimizes the error, between the estimation of the system's state and the actual system's state. Only the estimated state from the previous time step and the new measurements are needed to compute the new state estimate [Brown and Hwang 1997].

The challenge of using a Kalman filter lies in defining the linear stochastic difference equation governing the transition mechanics of the system from one state $x \in \mathfrak{R}^{n}$ to another.

$$
x_{k+1}=A_{k+1} x_{k}+B_{k+1} u_{k+1}+w_{k+1}
$$

with the measurement

$$
z_{k}=H_{k} x_{k}+v_{k}
$$

The $n$-by-n state transition matrix $A_{k+1}$ relates the state at the previous time step $\mathrm{k}$ to the state at the current step $\mathrm{k}+1$, in the absence of either a driving function or process noise. $\mathrm{B}_{\mathrm{k}+1}$ is an $\mathrm{n}$ by-m control input matrix, that relates $m-b y-1$ control vector $u_{k+1}$ to the state $\mathrm{x}_{\mathrm{k}} \cdot \mathrm{w}_{\mathrm{k}}$ is an n-by-1 system's noise vector with an n-by-n covariance matrix $\mathrm{Q}_{\mathrm{k}} \cdot p\left(w_{k}\right) \sim N\left(0, Q_{k}\right)$. Not all variables in the state are visible to the measuring instruments. The measurement vector $Z_{k}$ contains state variables that are measured by the instruments. $\mathrm{H}_{\mathrm{k}}$ is a j-by-n observation model matrix which maps the state $\mathrm{x}_{\mathrm{k}}$ into the measurement vector $\mathrm{z}_{\mathrm{k}} \cdot \mathrm{v}_{\mathrm{k}}$ is a measurement noise j-by-1 vector with covariance $\mathrm{R}_{\mathrm{k}} . p\left(v_{k}\right) \sim N\left(0, R_{k}\right)$.

The Discrete Kalman filter has two distinct phases that compute the estimate of the next system's state [Brown and Hwang 1997].

\section{Predict:}

Predict the state vector ahead:

$$
\hat{x}_{k+1}^{-}=A_{k+1} x_{k}+B_{k+1} u_{k+1}
$$

The $\hat{x}_{k+1}^{-}$is used as the future eye position coordinate for predicting eye movement trajectories.

Predict the error covariance matrix ahead:

$$
P_{k+1}^{-}=A_{k+1} P_{k} A_{k+1}^{T}+Q_{k+1}
$$

The predict phase uses the previous state estimate to predict the estimate of the next system's state.

\section{Update:}

Compute the Kalman gain:

$$
K_{k+1}=P_{k+1}^{-} H_{K+1}^{T}\left(H_{k+1} P_{k+1}^{-} H_{k+1}^{T}+R_{k+1}\right)^{-1} \quad \mathbf{3 1}
$$

Update the estimate of the state vector with a measurement $\mathrm{Z}_{\mathrm{k}+1}$ :

$$
\hat{x}_{k+1}=\hat{x}_{k+1}^{-}+K_{k+1}\left(z_{k+1}-H_{k+1} \hat{x}_{k+1}^{-}\right)
$$

Update the error covariance matrix:

$$
P_{k+1}=\left(I-K_{k+1} H_{k+1}\right) P_{k+1}^{-}
$$

It should be pointed out that the Kalman Filter maintains first two moments of the state distribution $E\left[x_{k}\right]=\hat{x}_{k}, E\left[\left(x_{k}-\right.\right.$ $\left.\left.\hat{x}_{k}\right)\left(x_{k}-\hat{x}_{k}\right)^{T}\right]=P_{k} \quad$ and $\quad p\left(x_{k} \mid z_{k}\right) \sim N\left(E\left[x_{k}\right], E\left[\left(x_{k}-\right.\right.\right.$ $\left.\left.\left.\hat{x}_{k}\right)\left(x_{k}-\hat{x}_{k}\right)^{T}\right]\right)=\mathrm{N}\left(\hat{x}_{k}, P_{k}\right)$. The choice of the Kalman gain $\mathrm{K}_{\mathrm{k}}$ 
minimizes error covariance matrix $\mathrm{P}_{\mathrm{k}}$. Kalman Filter framework assumes that $\mathrm{x}_{\mathrm{k}}, \mathrm{Z}_{\mathrm{k}}$ are normally distributed and $E\left[v_{k} v_{i}^{T}\right]=$ $\left\{\begin{array}{l}R_{k} i=k \\ 0 \quad i \neq k\end{array}, E\left[w_{k}, w_{i}^{T}\right]=\left\{\begin{array}{c}Q_{k} i=k \\ 0 \quad i \neq k\end{array}, E\left[w_{k} e_{i}^{T}\right]=0 \forall i, k\right.\right.$.

\subsection{TSKF \& OPKF}

Two State Kalman Filter (TSKF) employs the information about eye position and velocity. It has the capability to detect the onset and the parameters of a saccade by the mechanism described in our previous work [Komogortsev 2007b]. Once saccade is detected the Oculomotor Plant Kalman Filter (OPKF) uses mathematical equations describing the OPMM to define the transition matrix $A_{k+1}$, control matrix $B_{k+1}$ and control vector $u_{k+1}$. Figure 3 diagrammatically presents the difference between the TSKF and the OPKF models.

\subsubsection{State Vector}

Following values are selected to represent the state vector for both models:

$$
x_{k}=\left[\begin{array}{llllll}
x_{1}(k) & x_{2}(k) & x_{3}(k) & x_{4}(k) & x_{5}(k) & x_{6}(k)
\end{array}\right]^{T}
$$

$x_{1}(k)=\Delta \theta \quad-$ eye rotation, $\quad x_{2}(k)=\Delta \theta_{L T_{-} L R} \quad$ and $\quad x_{3}(k)=$ $\Delta \theta_{L T \_M R}$ - displacement of the length tension component for the lateral and medial recti respectively, $x_{4}(k)=\Delta \dot{\theta}-$ eye velocity, $x_{5}(k)=\hat{F}_{L R}$ and $x_{6}(k)=\hat{F}_{M R}$ active state tension for lateral and medial recti.

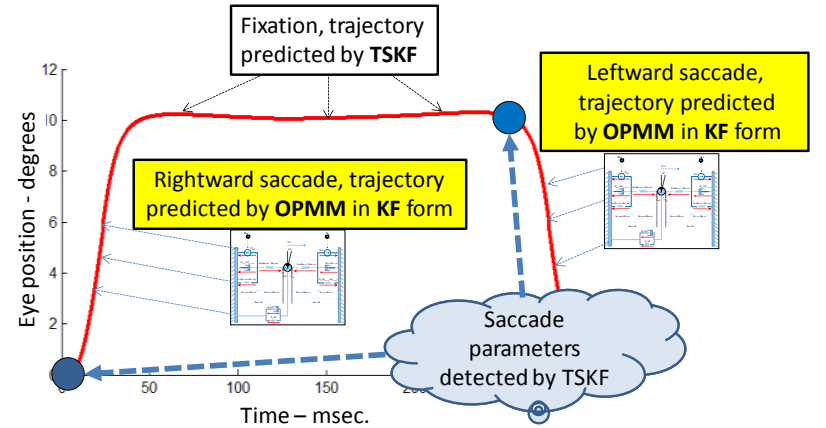

Figure 3. Eye movement prediction by the OPKF model i.e. all eye movements except saccades are predicted by TSKF and saccades are predicted by OPMM in the Kalman filter form. In case of pure TSKF model all eye movements would have been predicted by the TSKF.

\subsubsection{Transition matrix, control matrix, control vector: $A_{k}, B_{k}, u_{k}$.}

\section{TSKF}

TSKF uses only angular eye position $x_{1}(k)$ and velocity $x_{4}(k)$ parameters. In its simplest form horizontal eye movement dynamics can be represented by a differential equation $\dot{x}_{1}(k)=$ $x_{4}(k)$. Approximating derivative as $\dot{x}(k)=\frac{x(k+1)-x(k)}{\Delta t}$ the equation $\dot{x}_{1}(k)=x_{4}(k) \quad$ can be rewritten as: $x_{1}(k+1)=x_{1}(k)+x_{4}(k) \Delta t . \Delta t$ is the eye position sampling interval meaning that the samples $x_{i}(k+1)_{i}$ and $x_{i}(k)$ are $\Delta t$ seconds apart. The previous equation can be represented in form of Equation 29 with transition matrix $A_{k}=\left(\begin{array}{cccccc}1 & 0 & 0 & \Delta \mathrm{t} & 0 & 0 \\ 0 & 0 & 0 & 0 & 0 & 0 \\ 0 & 0 & 0 & 1 & 0 & 0 \\ 0 & 0 & 0 & 0 & 0 & 0 \\ 0 & 0 & 0 & 0 & 0 & 0\end{array}\right)$ and zero control matrix and control vector.

\section{OPKF}

Positive amplitude saccades:
Differential Equations 17,18,1920,22,23 derive $A_{k}, B_{k}, u_{k}$ for the OPKF model during saccades of the positive amplitude. First those equations are transformed into the following form:

$$
\begin{gathered}
\dot{x}_{1}(t)=x_{4}(t) \\
\dot{x}_{2}(t)=\frac{K_{S E}^{2}}{\left(K_{L T}+K_{S E}\right) \hat{B}_{A G}} x_{1}(t)-\frac{K_{S E}}{\hat{B}_{A G}} x_{2}(t)+\frac{K_{S E}}{\left(K_{L T}+K_{S E}\right) \hat{B}_{A G}} x_{5}(t) \\
\dot{x}_{3}(t)=\frac{K_{S E}^{2}}{\left(K_{L T}+K_{S E}\right) \hat{B}_{A N T}} x_{1}(t)-\frac{K_{S E}}{\hat{B}_{A N T}} x_{3}(t)-\frac{K_{S E}}{\left(K_{L T}+K_{S E}\right) \hat{B}_{A N T}} x_{6}(t) \\
\dot{x}_{4}(t)=-\frac{2 K_{S E}+K_{p}}{J} x_{1}(t)+\frac{K_{S E}}{J} x_{2}(t)+\frac{K_{S E}}{J} x_{3}(t)-\frac{B_{p}}{J} x_{4}(t) \\
\dot{x}_{5}(t)=\frac{N_{A G_{-} s a c}-x_{5}(t)}{\tau_{A G}} \\
\dot{x}_{6}(t)=\frac{N_{A N T_{-} s a c}-x_{6}(t)}{\tau_{A N T}}
\end{gathered}
$$

Second using the approximate definition of derivative as $\dot{x}(k)=$ $\frac{x(k+1)+x(k)}{\Delta \rho}\left(\Delta \rho\right.$ is the OPMM internal sampling clock $\left.^{1}\right)$ the transition matrix $A_{k}$, control matrix $B_{k}$, control vector $u_{k}$ are calculated. Transition matrix $A_{k}$ is the following: $\left(\begin{array}{cccccc}\Delta \rho \frac{K_{S E}^{2}}{\left(K_{L T}+K_{S E}\right) \hat{B}_{A G}} & \left(1-\Delta \rho \frac{K_{S E}}{\hat{B}_{A G}}\right) & 0 & 0 & \Delta \rho \frac{K_{S E}}{\left(K_{L T}+K_{S E}\right) \hat{B}_{A G}} & 0 \\ \Delta \rho \frac{K_{S E}^{2}}{\left(K_{L T}+K_{S E}\right) \hat{B}_{A N T}} & 0 & \left(1-\Delta \rho \frac{K_{S E}}{\hat{B}_{A N T}}\right) & 0 & 0 & -\Delta \rho \frac{K_{S E}}{\left(K_{L T}+K_{S E}\right) \hat{B}_{A N T}} \\ -\Delta \rho \frac{2 K_{S E}+K_{p}}{J} & \Delta \rho \frac{K_{S E}}{J} & \Delta \rho \frac{K_{S E}}{J} & 1-\Delta \rho \frac{B_{p}}{J} & 0 & 0 \\ 0 & 0 & 0 & 0 & \left(1-\frac{\Delta \rho}{\tau_{A G}}\right) & 0 \\ 0 & 0 & 0 & 0 & 0 & \left(1-\frac{\Delta \rho}{\tau_{A N T}}\right)\end{array}\right)$
$u_{k}=\left[\begin{array}{llllll}0 & 0 & 0 & 0 & \frac{\Delta \rho}{\tau_{A G}} N_{A G_{-} s a c} & \frac{\Delta \rho}{\tau_{A N T}} N_{A N T \_s a c}\end{array}\right]^{T}$ The control matrix $B_{k}$ is a $6 \times 6$ identity matrix. For more detailed calculations please look in [Komogortsev 2007c].

Negative amplitude saccades:

Differential Equations 17,18,23,24,25,26 derive $A_{k}, B_{k}$, $u_{k}$ for the OPKF model during saccades of the positive amplitude. First those equations are transformed into the following form:

$$
\begin{gathered}
\dot{x}_{1}(t)=x_{4}(t) \\
\dot{x}_{2}(t)=\frac{K_{S E}^{2}}{\left(K_{L T}+K_{S E}\right) \hat{B}_{A N T}} x_{1}(t)-\frac{K_{S E}}{\hat{B}_{A N T}} x_{2}(t)+\frac{K_{S E}}{\left(K_{L T}+K_{S E}\right) \hat{B}_{A N T}} x_{5}(t) \\
\dot{x}_{3}(t)=\frac{K_{S E}^{2}}{\left(K_{L T}+K_{S E}\right) \hat{B}_{A G}} x_{1}(t)-\frac{K_{S E}}{\hat{B}_{A G}} x_{3}(t)-\frac{K_{S E}}{\left(K_{L T}+K_{S E}\right) \hat{B}_{A G}} x_{6}(t) \\
\dot{x}_{4}(t)=-\frac{2 K_{S E}+K_{p}}{J} x_{1}(t)+\frac{K_{S E}}{J} x_{2}(t)+\frac{K_{S E}}{J} x_{3}(t)-\frac{B_{p}}{J} x_{4}(t) \\
\dot{x}_{5}(t)=\frac{N_{A N T \_s a c}-x_{5}(t)}{\tau_{A N T}} \\
\dot{x}_{6}(t)=\frac{N_{A G_{-} s a c}-x_{6}(t)}{\tau_{A G}}
\end{gathered}
$$

Second the transition matrix $A_{k}$, control matrix $B_{k}$, control vect or $\mathrm{u}_{\mathrm{k}}$ are calculated. Transition matrix $A_{k}$ is the following:

$$
\begin{aligned}
& \left(\begin{array}{cccccc}
\Delta \rho \frac{K_{S E}^{2}}{\left(K_{L T}+K_{S E} \hat{B}_{A N T}\right.} & \left(1-\Delta \rho \frac{K_{S E}}{\hat{B}_{A N T}}\right) & 0 & 0 & \Delta \rho \frac{K_{S E}}{\left(K_{L T}+K_{S E}\right) \hat{B}_{A N T}} & 0 \\
\Delta \rho \frac{K_{S E}^{E}}{\left(K_{L T}+K_{S E E}\right)_{A G}} & 0 & \left(1-\Delta \rho \frac{K_{S E}}{\widehat{B}_{A G}}\right) & 0 & 0 & -\Delta \rho \frac{K_{S E}}{\left(K_{L T}+K_{S E}\right) \hat{B}_{A G}} \\
-\Delta \rho \frac{2 K_{S E}+K_{p}}{J} & \Delta \rho \frac{K_{S E}}{J} & \Delta \rho \frac{K_{S E}}{J} & 1-\Delta \rho \frac{B_{p}}{J} & 0 & 0 \\
0 & 0 & 0 & 0 & \left(1-\frac{\Delta \rho}{\tau_{A N T}}\right) & 0 \\
0 & 0 & 0 & 0 & 0 & \left(1-\frac{\Delta \rho}{\tau_{A G}}\right)
\end{array}\right) \\
& u_{k}=\left[\begin{array}{llllll}
0 & 0 & 0 & 0 & \frac{\Delta \rho}{\tau_{A N T}} N_{A N T_{-} s a c} & \frac{\Delta \rho}{\tau_{A G}} N_{A G_{-} s a c}
\end{array}\right]^{T}
\end{aligned}
$$

1 The Oculomotor Plant Mechanical Model generates best results with internal clock $\Delta \rho=0.001 \mathrm{sec}$. due to neuronal control signal changing on the millisecond level. If the internal clock is larger than $1 \mathrm{msec}$. the difference between the actual eye movement trajectory and the trajectory generated by oculomotor plant mechanical model will increase. 
The control matrix $B_{k}$ is a $6 \times 6$ identity matrix.

\subsubsection{Measurement vector, observation matrix: $z_{k}, H_{k}$.}

The eye position measurement device is an eye tracker. An eye tracker reports horizontal and vertical eye position coordinates with a time stamp. In this paper only horizontal component of the recorded eye movements is considered, thus measurement vector $\mathrm{Z}_{\mathrm{k}}$ is a scalar that represents horizontal eye coordinate recorded by the eye tracker at the time $\mathrm{k}$.

The angular eye position is the only variable that is observed, making observation matrix $H_{k}=\left[\begin{array}{llllll}1 & 0 & 0 & 0 & 0 & 0\end{array}\right]$.

\subsubsection{Measurement noise covariance matrix, system's noise covariance matrix: $R_{k}, Q_{k}$.}

By definition the covariance matrix for the measurement noise is $R_{k}=E\left[\left(v_{k}-E\left(v_{k}\right)\right)\left(v_{k}-E\left(v_{k}\right)\right)^{T}\right]$. Because only eye position is measured $v_{k}$ is a scalar $R_{k}=\operatorname{VAR}\left[v_{k}\right]=\delta_{v}^{2}$, where $\delta_{v}$ is the standard deviation of the measurement noise. We assume that the standard deviation of the measurement noise relates to the accuracy of the eye tracker and is bounded by one degree of the visual angle. Therefore $\delta_{v}$ was conservatively set to $1^{\circ}$. In case when the eye tracker fails to detect eye position coordinates the standard deviation of measurement noise is assigned to be

$$
\delta_{v}=120^{\circ}
$$

The value of $120^{\circ}$ is chosen empirically, allowing Kalman filter to "trust" more predicted eye position coordinate $\hat{x}_{k}^{-}$.

By definition system's noise covariance matrix is $Q_{k}=$ $E\left[\left(w_{k}-E\left(w_{k}\right)\right)\left(w_{k}-E\left(w_{k}\right)\right)^{T}\right]$, where $w_{k}$ is a $1 \times 6$ system's noise vector

$w_{k}=\left[\begin{array}{llllll}w_{1}(k) & w_{2}(k) & w_{3}(k) & w_{4}(k) & w_{5}(k) & w_{6}(k)\end{array}\right]^{T}$. We assume that variables $w_{i}(k)$ are uncorrelated between each other i.e. $E\left[\left(w_{m}(k) w_{n}(k)\right]=E\left[\left(w_{m}(k)\right] E\left[w_{n}(k)\right]\right.\right.$ for all $n \neq m$. This assumption generates following system's noise covariance matrix: $Q_{k}=\left[\begin{array}{ccc}\delta_{1}^{2} & \ldots & 0 \\ \ldots & \ldots & \ldots \\ 0 & \ldots & \delta_{6}^{2}\end{array}\right]$. Here $\delta_{1}^{2}, \ldots, \delta_{6}^{2}$ are variances of variables $w_{i}(k)$. We assume that the standard deviation of the eye position noise $w_{1}(k)$ is connected to the characteristics of the eye fixation movement. This is done with the assumption that eye fixation is the most common type of the eye movement. Each eye fixation consists of three basic eye-sub-movements: drift, small involuntary saccades and tremor [Yarbus 1967]. Among those three, involuntary saccades have the highest amplitude - around half degree of the visual angle, therefore we conservatively set $\delta_{1}$ to $1^{\circ}$. Standard deviation values for other variables are hard to assess, but the following values performed well in the simulation tests: $\delta_{2}=\delta_{3}=1^{\circ}$ degree, $\delta_{4}=1 \%$ sec., $\delta_{5}=\delta_{6}=1$ gram.

\subsubsection{Initial values for state vector and error covariance matrices: $x_{0}, P_{0}$.}

The TSKF model uses following initial state vector $x_{0}=$ $\left[\begin{array}{llllll}x_{1}(0) & x_{2}(0) & x_{3}(0) & x_{4}(0) & x_{5}(0) & x_{6}(0)\end{array}\right]^{T} \quad$ Here last measured horizontal eye position coordinate is assigned to $x_{1}(0)$ and $x_{2}(0)=x_{3}(0)=x_{4}(0)=x_{5}(0)=x_{6}(0)=0$.

The OPKF uses the following values at the beginning of each saccades: $x_{1}(0)$ - horizontal saccade onset eye position; $x_{2}(0)=$ $x_{1}(0)+5.6^{\circ}$ - initial displacement of the length of the lateral rectus length tension component; $x_{3}(0)=x_{1}(0)-5.6^{\circ}$ - initial displacement of the length of the medial rectus length tension component; $x_{4}(0)=0$ - initial value of the eye velocity; initial active state tension for the lateral rectus is calculated as $x_{5}(0)=$ $N_{A G_{\text {_sac_start }}}\left(x_{1}(0)\right) ; x_{6}(0)=N_{A N T_{-} \text {sac_start }}\left(x_{1}(0)\right)$.

\section{Methodology}

\subsection{Equipment \& Test Media}

Eye movement prediction models were tested with a Tobii 1750 Eye Tracker and the following video clips. Car: This video shows a moving car. It was taken from a security camera view point in the university's parking lot. The visible size of the car is approximately one fifth of the screen. The car moves slowly. Several pedestrians and distant cars appeared on the background several times, often capturing the attention of the subject. Shamu: This video captures a spotlighted, evening performance of Shamu at Sea World. This video consists of several moving objects: Shamu, the trainer, and the audience. Each of them is moving at different speeds during various periods of time. Airplanes: This video depicts a performance of the Blue Angels on Lake Erie. The flight formation of supersonic planes changes rapidly as does their flight speeds. The camera movements were rapid zoom and panning. All three videos had a resolution of $720 \times 480$ pixels, presented with a frame-rate of $30 \mathrm{fps}$, and were 1 minute long. The videos Participants \& Evaluation

The subject pool consisted of 21 volunteers of both genders and mixed ethnicities, aged 20-40 with normal, corrected and uncorrected vision. The subjects were instructed to watch the video clips in any way they wanted.

For performance comparison both the TSKF and the OPKF eye movement prediction models were implemented in MATLAB. The horizontal movement component of the right eye was analyzed offline. Prediction interval for both models was $20 \mathrm{msec}$.

\subsection{Detection of Basic Eye Movement Types}

The IV-T model is used for the eye movement detection [Salvucci and Goldberg 2000]. The detection criteria is as follows: eye fixation is detected when the eye speed does not exceed $20 \% \mathrm{sec}$ and this velocity pattern lasts for at least $100 \mathrm{msec}$., which is considered to be the minimum duration interval for an eye fixation. A saccade is detected when the eye velocity exceeds $300 \%$ sec. Pursuits are detected when the eye velocity is in the $20-300 \% / \mathrm{sec}$ range.

\subsection{Eye Movement Prediction Accuracy Metric}

The root mean squared error (RMSE) between the predicted $\hat{x}_{1}^{-}(k)$, Equation 29, and measured $\mathrm{z}_{\mathrm{k}}$, Equation 28, eye position coordinate represents the accuracy of an eye movement prediction. $R M S E=\sqrt{\sum_{k=i}^{j} \frac{\left(\widehat{x}_{1}^{-}(k)-z_{k}\right)^{2}}{j-i}}$. The ideal eye movement prediction model will have the RMSE of $0^{\circ}$. The percentage improvement in prediction accuracy (reduction of the RMSE) between various eye movement prediction models was calculated by the following formula: $\Lambda=100 \frac{R M S E_{\text {Model___ }}-R M S E_{\text {Model__2 }}}{R E_{S_{0}}}$.

\section{Results}

\subsection{Test Video Set Performance}

Fixations: Average fixation duration was approximately 130 msec., deviating 30-40 msec. from the mean. The percentage of the fixations in the eye movement trace was $12-15 \%$. Saccades: Saccade amplitude was the highest for the "Car" $-17^{\circ} .16^{\circ}$ for the "Shamu" video and $14^{\circ}$ for the "Airplanes" video. The percentage 
of saccades in the eye movement trace was 3-4\%. Pursuits: The percentage of saccades in the eye movement trace was $76-78 \%$. Not Reported:The Not Reported category is the percentage of eye position samples for which the eye tracker failed to report the eye position coordinates. The amount of such samples on average was between $5-8 \%$.

\subsection{Eye Movement Prediction Results}

\subsubsection{Fixations \& Pursuits}

We assume that the main source of the prediction error during fixations comes from the fact that the eye is not absolutely stationary during a fixation; there are various types of submovements during a fixation i.e. tremor, drift and involuntary saccades with amplitudes of up to $0.5^{\circ}$ [Yarbus 1967]. The TSKF and the OPKF models produced an RMSE in the range of 0.94$0.97^{\circ}$ during eye fixations and $2.65-3.12^{\circ}$ during pursuits. This result indicates that the Two State Kalman Filter modeling the Human Visual System through just eye position and velocity can moderately predict the eye movement trajectory during eye fixations and pursuits.

\subsubsection{Saccades}

\begin{tabular}{|c|c|c|c|}
\hline Saccades & $R M S E-T S K F$ & $R M S E-O P K F$ & Accuracy imp. \\
\hline "Car" & 14.50 & 10.55 & $27 \%$ \\
\hline "Shamu" & 13.52 & 9.98 & $28 \%$ \\
\hline "Airplanes" & 12.13 & 9.99 & $18 \%$ \\
\hline
\end{tabular}

The RMSE was the highest for the "Car" video presumably due to highest saccade amplitudes. The RMSE reported for the "Shamu" video was lower, due to the lower saccade amplitudes, but the recorded accuracy improvement was the highest. In case of the "Airplanes" video the improvement in the accuracy prediction was the smallest due to the smaller saccade amplitudes exhibited by subjects during this video. The accuracy improvement achieved by the employment of the OPMM is most effective for saccades of large amplitudes. A valid question to ask is "What are the factors limiting further improvements in the prediction accuracy achieved by the OPKF model?" At least one of those factors is tied to how soon a saccade can be detected by the TSKF. Given an eye position sampling frequency of $50 \mathrm{~Hz}$ the experiments indicate that the chi square test detects a saccade usually with the first measured eye position sample that is available after the beginning of the saccade. In the setup presented in this paper, $20 \mathrm{msec}$. of trajectory from the beginning of detected saccade is predicted by the TSKF. This limits the improvement achieved by the OPKF to the remaining part of the saccade trajectory. Results indicated that the TSKF is not very accurate in predicting saccadic eye movements. We believe higher eye position sampling frequency will allow saccade parameters to be estimated sooner and will result in more accurate prediction of the saccade trajectories by the OPKF.

\subsubsection{All Eye Movements}

The "All" category calculated the overall performance of each prediction model for all eye movements by calculating the RMSE for all eye position samples except "Not Reported" category.

\begin{tabular}{|c|c|c|c|}
\hline All & RMSE - TSKF & RMSE - OPKF & Accuracy imp. \\
\hline "Car" & 4.13 & 3.12 & $13 \%$ \\
\hline "Shamu" & 3.82 & 3.06 & $12 \%$ \\
\hline "Airplanes" & 3.29 & 2.65 & $7 \%$ \\
\hline
\end{tabular}

The "Car" video had the highest prediction error overall, but prediction accuracy improvement was the largest as well. The "Airplanes" video with smaller saccade amplitudes had the smallest accuracy improvement.

\section{Conclusion}

Eye tracking technology can successfully enhance already existing interaction methods. There are several issues that are needed to be resolved before this can happen. One of these issues is related to the fact that eye tracking equipment and interactive environments in general possess various types of delays. The delays can be compensated by an accurate eye movement prediction. In this paper we have designed an Oculomotor Plant Kalman Filter that provides a continuous accurate eye movement prediction. A key factor allowing achievement of a high degree of prediction accuracy is a mechanical model of oculomotor plant transformed into a Kalman filter form that is employed during saccades. Such an approach improves eye movement prediction accuracy during saccades by $18-28 \%$ and by $7-13 \%$ overall. These results were achieved for $20 \mathrm{msec}$. prediction range, 21 subjects and passed the statistical significance test with the level of 0.01 .

\section{References}

Bahill, A. T. (1980). Development, validation and sensitivity analyses of human eye movement models. CRC Critical Reviews in Bioengineering , 4, 311-355.

Brown, R., \& Hwang, P. (1997). Introduction to Random Signals and Applied Kalman Filtering (3rd Edition ed.). New York: John Wiley and Sons.

Collins, C. C. (1975). The human oculomotor control system. (F. Lennerstrand, \& P. Bach-Rita, Eds.) Basic Mechanisms of Ocular Motility and Their Applications, 145-180.

Duchowski, A. T. (2003). Eye Tracking Methodology: Theory and Practic. London: Springer-Verlag.

Jacob, K. R. (1995). Eye tracking in advanced inteface design. New York: Oxford University Press, Inc.

Komogortsev, O. V. (2007a). World of Warcraft Percept Interface. World of Warcraft Percept Interface

Komogortsev, O. V., \& Khan, J. (2007b). Kalman Filtering in the Design of Eye-Gaze-Guided Computer Interfaces. 12th International Conference on Human-Computer Interaction (HCI 2007), (pp. 1-10). Beijing, China.

Komogortsev, O. V. (2007c). Eye Movement Prediction by Oculomotor Plant Modeling with Kalman Filter. Ph.D. Dissertation . Kent, OH, USA: Kent State University.

Komogortsev, O. (2007d). Perceptual Test Video Set. from www.cs.txstate.edu/ ok11/videosetpercept.htm

Murtagh, F., Farid, M., \& Starck, J. L. (2002). Computer display control and interaction using eye-gaze. Journal of the Society for Information Display, 10(3), 289-293.

Robinson, D. A., Omeara, D. M., Scott, A. B., \& Collins, C. C. (1969). Mechanical components of human eye movements. Journal of Applied Physiology, 548-553.

Salvucci, D. D., \& Goldberg, J. H. (2000). Identifying fixations and saccades in eye tracking protocols. Eye Tracking Research and Applications Symposium (pp. 71-78).

Sauter, D., J., M. B., Di Renzo, N., \& Vomscheid, C. (1991). Analysis of eye tracking movements using innovations generated by a Kalman filter. Med. Biol. Eng. Comput. , 63-69.

Sparks, D. L. (2002). The brainstem control of saccadic eye movements. Nat. Rev. Neurosci. , 3(12), 952-964.

Wilkie, D. R. (1976). Muscle. Studies in Biology, 11. Yarbus, L. (1967). Eye Movements and Vision. Moscow: Institute for Problems of Information Transmission Academy of Sciences of the USSR. 


\section{Eye Movement Prediction by Kalman Filter with Integrated Linear Horizontal Oculomotor Plant Mechanical Model Komogortsev and Khan}

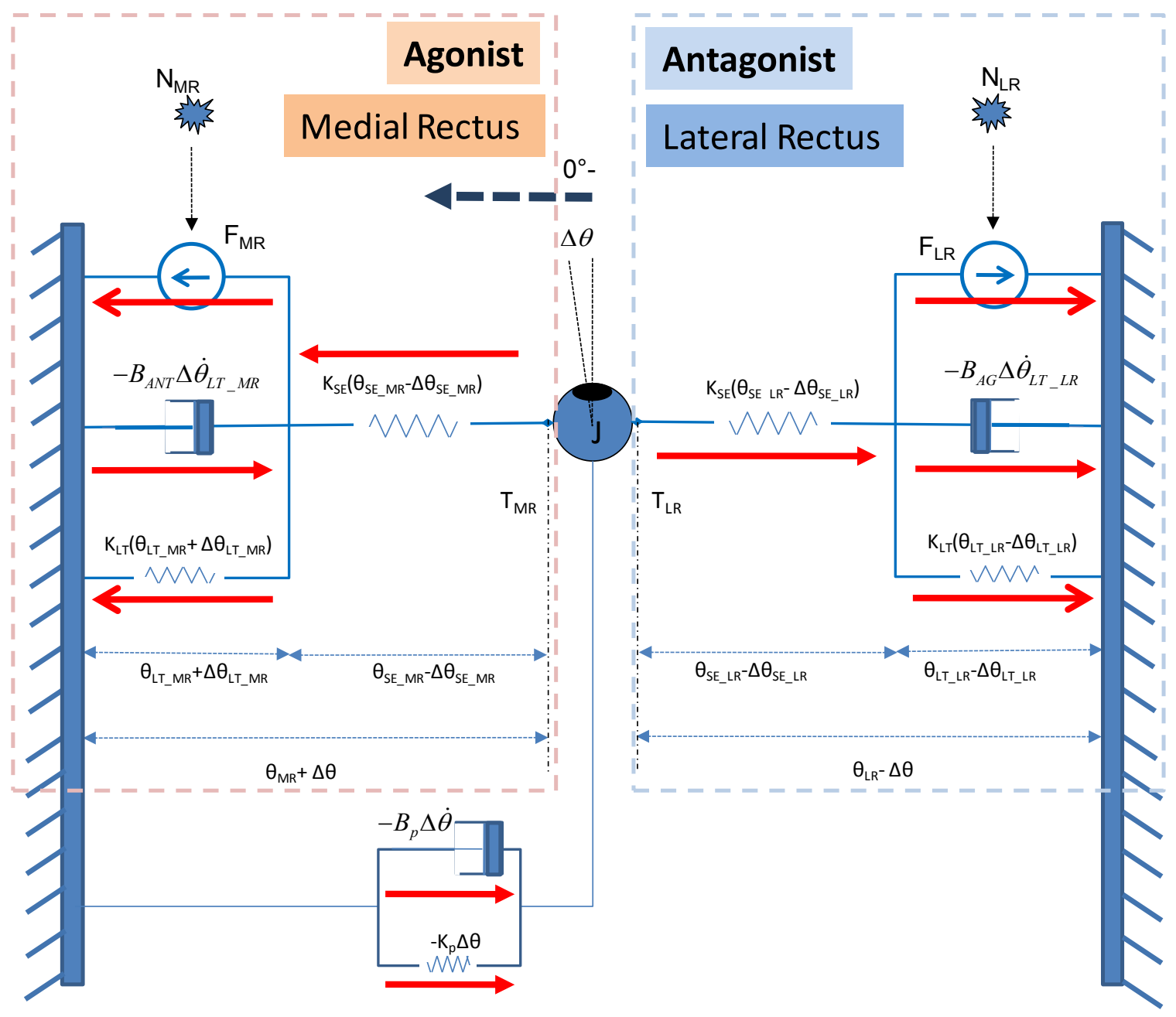

Figure 2. The Oculomotor Plant Mechanical Model employed during saccades of negative amplitude. 